\title{
Measurement of magnetic noise in magnetoimpedance sensing element
}

\author{
Anatoliy S. Antonov ${ }^{1, *}$, Nikita A. Buznikov ${ }^{2}$, Timur T. Sultan-Zade ${ }^{1}$, and Alexey A. Shkliaev ${ }^{3}$ \\ ${ }^{1}$ IMPEDANCE JSC, 124527 Zelenograd, Moscow, Russia \\ ${ }^{2}$ Scientific \& Research Institute of Natural Gases and Gas Technologies - Gazprom VNIIGAZ, \\ 142717 Razvilka, Leninsky District, Moscow Region, Russia \\ ${ }^{3}$ Decima LLC, 124498, Zelenograd, Moscow, Russia
}

\begin{abstract}
A method for measurement of magnetic noise in magnetoimpedance sensing element is proposed. Glass-coated Co-based amorphous microwires with a slightly negative magnetostriction were used as the sensing element. The operation principle of the sensing element was based on the nonlinear off-diagonal magnetoimpedance, when field dependent higher harmonic components appeared in voltage in the pick-up coil wound around the microwire. The magnetic noise for the second harmonic in the pick-up coil voltage was studied. The dependences of the magnetic noise and the signal-to-noise ratio in the sensitive element on the current amplitude were analyzed. The noise performance of the second harmonic in the pick-up coil voltage was compared to that for a flux-gate sensing element.
\end{abstract}

\section{Introduction}

The magnetoimpedance (MI) implies a strong dependence of the impedance of a magnetic conductor on an external magnetic field. The MI is attracted considerable attention due to its potential for magnetic sensing applications (see, for example, [1-4]). The MI effect is usually observed as a change of the voltage across the magnetic sample ends related to the external field variation. Another approach to detect the field dependent signal consists in the measurement of the voltage in the pick-up coil wound around the magnetic conductor [5]. The latter effect is referred to as the off-diagonal MI.

At relatively high amplitudes of the excitation current, the generation of higher harmonics in voltage has been reported (the so-called nonlinear MI) [6-12]. The origin of the nonlinear voltage response is ascribed to the magnetization reversal of a conductor under the magnetic field generated by the current. The nonlinear MI may be promising for applications, since higher harmonics are very sensitive to the external field.

One of the most important features of magnetic sensors is the capability to detect the external field with high accuracy. In this connection, it is of importance to achieve low noise level. The noise performance in different sensors based on the linear MI effect has been studied previously (see, for example, [13-17]). A model to calculate the intrinsic magnetic noise of MI sensing element has been proposed in [18], and the contributions of the sensing element and electronic circuits to the noise have been analyzed in detail $[14,15]$. However, precise separation of noise sources is a difficult task.
In this work, we present a direct method for the magnetic noise measurement in MI sensing element. The noise in the sensing element was measured by using well-known method of the transferring of low-frequency range to the high-frequency range based on the principle of the heterodyne transformation. In contrast to the previous studies, we investigated the nonlinear off-diagonal MI [12], and the second harmonic amplitude in the pickup coil voltage was used for the analysis of the magnetic noise performance. The magnetic noise in the sensitive element was analyzed as a function of the excitation current amplitude. The magnetic noise in the nonlinear off-diagonal MI sensing element was compared to the noise in a flux-gate sensing element.

\section{Experimental}

Glass-coated $\mathrm{CoFeSiB}$ amorphous microwires with a slightly negative magnetostriction were used as a MI sensing element. The microwires fabricated by Taylor-Ulitovsky method had the amorphous core diameter of $16 \mu \mathrm{m}$, and the glass coating thickness was $1 \mu \mathrm{m}$. The studied samples had the length of $6 \mathrm{~mm}$.

The MI effect in the microwire was measured by means of a conventional procedure using Agilent Impedance Analyzer 4294A. To study the nonlinear offdiagonal MI, the pick-up coil was wound around the microwire. The coil had 125 turns and its inner diameter was of $2 \mathrm{~mm}$. In experiments, the current amplitude was high enough to induce the magnetization reversal process in the microwire and was changed up to $30 \mathrm{~mA}$. The pick-up coil voltage was measured by means of Rohde\&Schwarz FSH8 Spectrum Analyzer. 


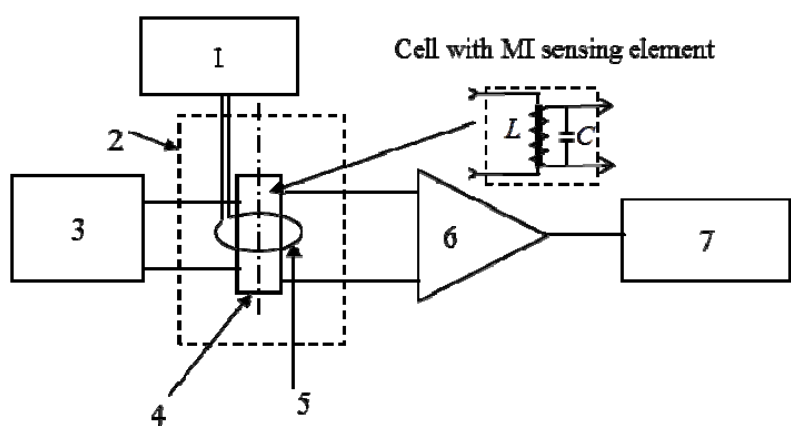

Fig. 1. A schematic of the experimental setup for noise measurements. 1, low-frequency generator; 2, magnetic shield; 3, high-frequency generator; 4 , sensing element; 5 , calibrated coil; 6, low-noise amplifier; 7, FSH8 Spectrum Analyzer.

The magnetic noise in the sensing element was measured by means of the frequency demodulation method. The schematic of the experimental setup is shown in Fig. 1. The setup allows one to study the different principles of the field detection in the sensing element, namely, the MI effect, off-diagonal MI and nonlinear off-diagonal MI. In this work, we focus on the latter effect and analyze the noise for the second harmonic in the pick-up coil voltage frequency spectrum.

The frequency of the excitation current was about $1 \mathrm{MHz}$. The pick-up coil wound around the microwire can be included in the resonant circuit with a capacitor, which was tuned to the second harmonic frequency. The studied microwire was placed into the magnetic shield in order to reduce external noise. The magnetic shield provided the protection at the level of $40 \mathrm{~dB}$. The low-noise amplifier was used to match with the low-impedance input of FSH8 Spectrum Analyzer.

\section{Results and discussion}

Fig. 2 shows the field dependence of the microwire impedance at the frequency $f_{0}=2 \mathrm{MHz}$. The measurements were carried out for the excitation current amplitude of $1 \mathrm{~mA}$. Within this frequency range, the imaginary part of the impedance has the strong dependence on the external field. The dependence has a typical two-peak behavior, with the maxima being at the field of the order of \pm 1 Oe.

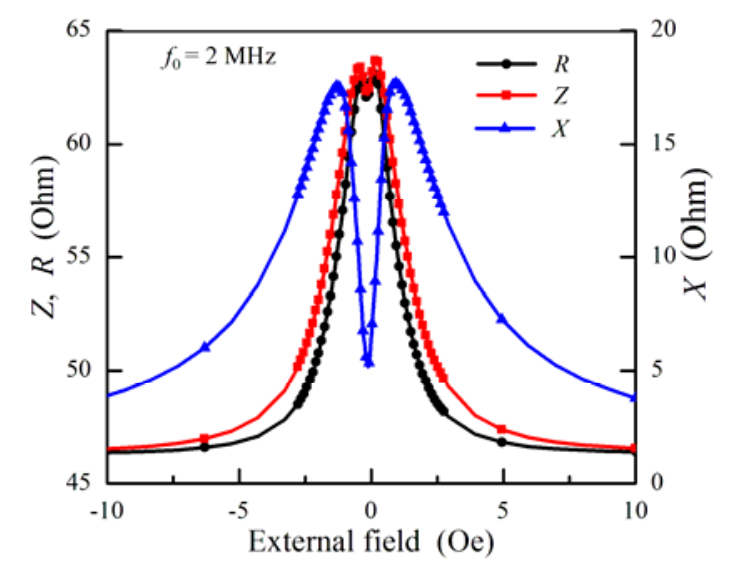

Fig. 2. Field dependences of the microwire impedance $Z$, its real $R$ and imaginary $X$ parts.

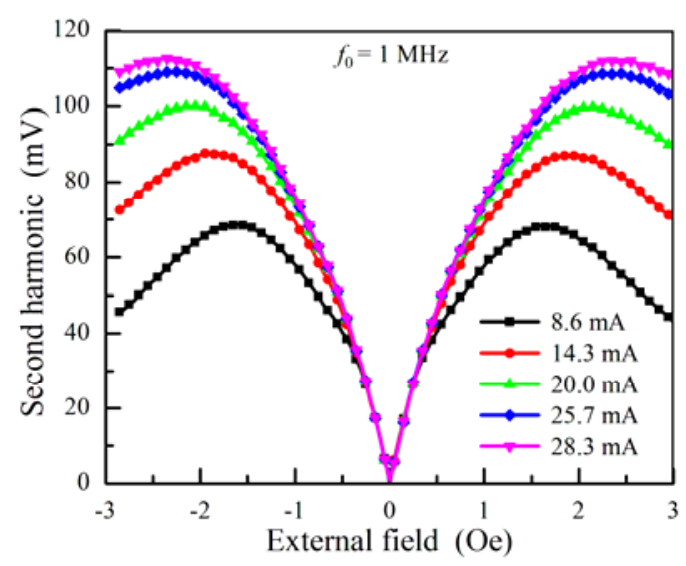

Fig. 3. Field dependence of the second harmonic amplitude in the pick-up coil voltage at different current amplitudes.

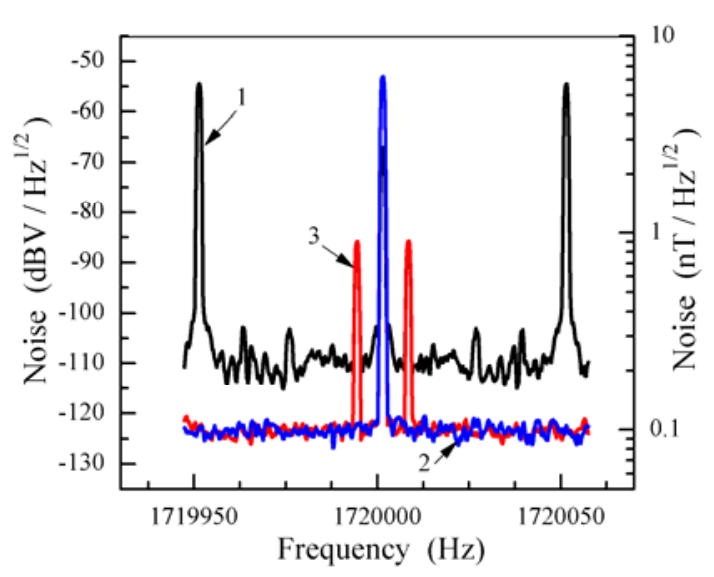

Fig. 4. An example of measured frequency spectrum of magnetic noise. Curve 1 , noise measured in the absence of the magnetic shield; 2, noise measured in the presence of the magnetic shield for signal without modulation; 3 , noise measured in the presence of the magnetic shield for signal with modulation.

At relatively high current amplitudes, the pick-up coil voltage response becomes nonlinear and consists of a number of harmonics due to the magnetization reversal in the microwire. It was shown previously that the second harmonic is dominant one in the pick-up voltage within a wide range of current amplitudes [12]. The measured field dependence of the second harmonic amplitude is presented in Fig. 3 for different values of the current amplitude for the excitation frequency $f_{0}=1 \mathrm{MHz}$. The second harmonic exhibited high sensitivity to the external field of the order of $50 \mathrm{mV} / \mathrm{Oe}$.

A typical frequency spectrum obtained in the measurements of the noise in the sensing element is presented in Fig. 4. The frequency range was selected as $\pm 55 \mathrm{~Hz}$ with respect to the second harmonic frequency $2 f_{0}=1.72 \mathrm{MHz}$. Three curves in Fig. 4 correspond to the measurements of the noise without the magnetic shield and in the presence of the shield without modulation and with modulation by a signal of the frequency of $7 \mathrm{~Hz}$. As a result of the modulation of the signal from the pick-up coil, two satellite peaks appeared in the noise frequency spectrum. The satellite peaks were symmetric with respect to the second harmonic frequency $2 f_{0}$ and differed from that at $7 \mathrm{~Hz}$. 
The amplitude of the pick-up coil signal in the units of nT was determined using the calibrating coil with an additional measurement by means of a magnetometer. A sinusoidal signal of the frequency of $7 \mathrm{~Hz}$ with the amplitude of $60 \mathrm{nT}$ was used. Using the calibration, the equivalent magnetic noise was recalculated in the units of $\mathrm{nT} / \mathrm{Hz}^{1 / 2}$.

Shown in Fig. 5 are the current amplitude dependences of the magnetic noise in the sensitive element based on the nonlinear off-diagonal MI and the signal-tonoise ratio for the excitation frequency of $0.86 \mathrm{MHz}$. The results are presented for both the resonant and nonresonant modes. In the resonant mode, the pick-up coil was connected to the resonance circuit tuned to the frequency $2 f_{0}=1.72 \mathrm{MHz}$. It follows from Fig. 5 that the magnetic noise and the signal-to-noise ratio depended slightly on the mode of the measurements. Note that the use of the capacitor in the circuit of the output signal can be of significance for applications, since it allows one to increase the transformation coefficient taking into account the resonance circuit quality factor. In the experiment, the quality factor was of the order of 10 , which allows one to obtain the transformation coefficient at the level of $1 \mathrm{~V} / \mathrm{Oe}$. As a result, the requirements to the primary amplifier of electronic circuit can be reduced for the same signal-to-noise ratio.

It follows from Fig. 5 (a) that the magnetic noise increased sharply for the range of current amplitudes 5-7 mA. The range corresponds to the circular magnetic field at the microwire surface of $1.25-1.75 \mathrm{Oe}$, when the
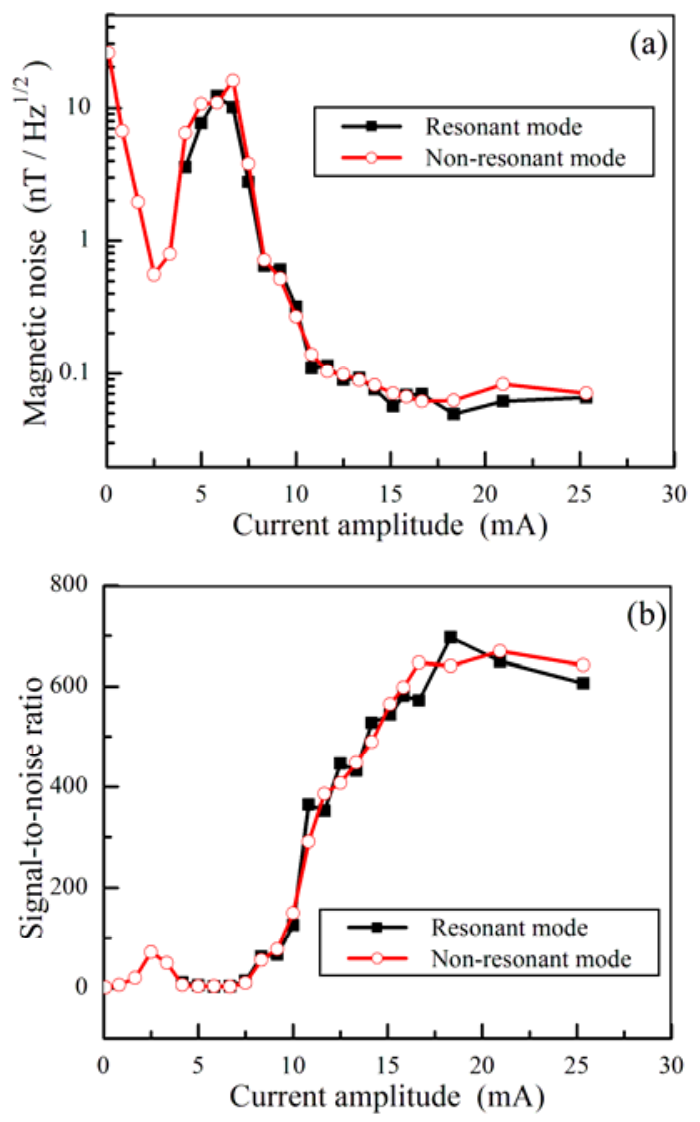

Fig. 5. Magnetic noise (a) and signal-to-noise ratio (b) versus current amplitude at $f_{0}=0.86 \mathrm{MHz}$.

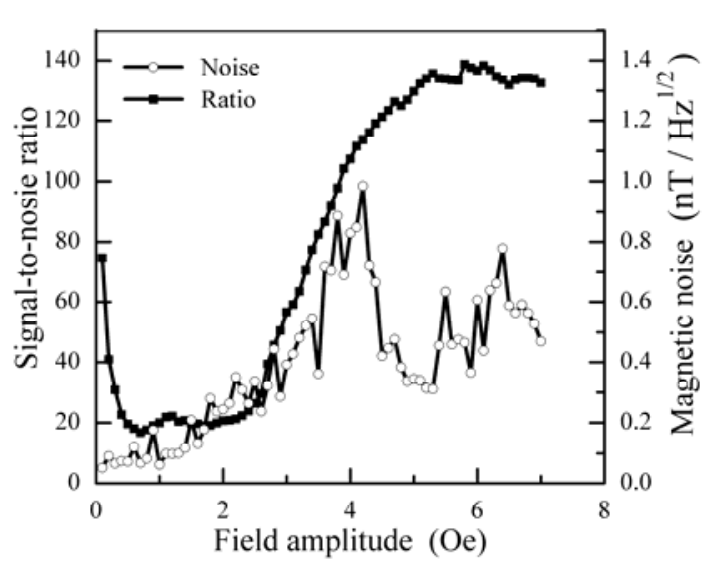

Fig. 6. The dependences of the magnetic noise and signal-tonoise ratio on the amplitude of the excitation magnetic field at $f_{0}=0.86 \mathrm{MHz}$ for the flux-gate sensing element.

transition of the pick-up coil voltage response to the nonlinear mode takes place. With a further increase of the current amplitude, the magnetic noise decreased and attained the level of $60 \mathrm{pT} / \mathrm{Hz}^{1 / 2}$ at the current amplitude exceeding $15 \mathrm{~mA}$. For the studied microwire, this range of the current amplitude seems to be optimal from the point of view of the signal-to-noise ratio.

Fig. 6 shows the results of the experimental study of the magnetic noise for the fluxgate configuration. The experiments were carried out for the same amorphous microwires. To obtain the signal with higher amplitude the volume of the sensing element was increased using twelve parallel microwires with length of $6 \mathrm{~mm}$. For the measurements, the same pick-up coil having 125 turns, inner diameter of $2 \mathrm{~mm}$ and length of $6 \mathrm{~mm}$ was used.

It follows from Fig. 6 that the magnetic noise for the flux-gate sensitive element attained maximum at the excitation field amplitude of the order of 4 Oe. This value is approximately two times higher than the amplitude of the threshold magnetic field for the sensitive element based on the nonlinear off-diagonal MI. When the field amplitude exceeded $5 \mathrm{Oe}$, the signal-to-noise ratio stabilized at the level of 140 . The magnetic noise for the flux-gate sensitive element was approximately 4.5 times higher than that for the nonlinear off-diagonal MI element, although the total length of the flux-gate element was twelve times higher.

This difference may be due to several reasons. Although the detailed explanation is beyond the scope of this work, note that the main reason of the difference in the magnetic noise level can be related to the principle of the excitation of the sensing element. In the flux-gate sensing element, the magnetization reversal appears due to the longitudinal field, and the alternating magnetic fields in the primary and output circuits have the collinear components. For the sensing element based on the nonlinear off-diagonal MI, the magnetization reversal is induced by the transverse magnetic field. The primary circuit forms the structure orthogonal to the output circuit, and there are no parallel components of the alternating magnetic field in these circuits. This fact results in the noise in the primary circuit of the sensing element is not transformed to the noise in the output circuit. 


\section{Conclusion}

A direct method for the magnetic noise measurement in the MI sensing element is proposed. The method allows one to study the magnetic noise in the sensing element for different principles of the field detection. The advantage of the method is that the signal and noise can be measured in a single experiment. In addition, the method allows one to avoid the noise of the electronic circuit forming the detected signal. The noise in the sensing element based on the detection of the second harmonic in the nonlinear off-diagonal MI response was investigated. The dependences of the magnetic noise and the signal-to-noise ratio in the sensitive element on the current amplitude were analyzed. A minimum noise level for the studied sensing elements was about $60 \mathrm{pT} / \mathrm{Hz}^{1 / 2}$ in the limit of low frequencies (at $1 \mathrm{~Hz}$ ). It was demonstrated that the magnetic noise in the nonlinear offdiagonal MI sensing element is significantly less than the noise in the flux-gate for a comparable size of the sensing element.

\section{References}

1. M. Vázquez, J. Magn. Magn. Mater. 226-230, 693 (2001)

2. M. Knobel, K.R. Pirota, J. Magn. Magn. Mater. 242-245, 33 (2002)

3. L. Kraus, Sens. Actuators A 106, 187 (2003)

4. M.-H. Phan, H.-X. Peng, Prog. Mater. Sci. 53, 323 (2008)

5. A.S. Antonov, I.T. Iakubov, A.N. Lagarkov, J. Magn. Magn. Mater. 187, 252 (1998)
6. R.S. Beach, N. Smith, C.L. Platt, F. Jeffers, A.E. Berkowitz, Appl. Phys. Lett. 68, 2753 (1996)

7. A. Yelon, M. Britel, D. Ménard, P. Ciureanu, Physica A 241, 439 (1997)

8. S. Iida, O. Ishii, S. Kambe, Jpn. J. Appl. Phys. 37, L869 (1998)

9. P. Aragoneses, A.P. Zhukov, J. Gonzalez, J.M. Blanco, L. Dominguez, Sens. Actuators A 81, $86(2000)$

10. C. Gómez-Polo, M. Knobel, K.R. Pirota, M. Vázquez, Physica B 299, 322 (2001)

11. G.V. Kurlyandskaya, H. Yakabchuk, E. Kisker, N.G. Bebenin, H. García-Miquel, M. Vázquez, V.O. Vas'kovskiy, J. Appl. Phys. 90, 6280 (2001)

12. A.S. Antonov, N.A. Buznikov, I.T. Iakubov, A.N. Lagarkov, A.L. Rakhmanov, J. Phys. D: Appl. Phys. 34, 752 (2001)

13. S. Yabukami, H. Mawatari, N. Horikoshi, Y. Murayama, T. Ozawa, K. Ishiyama, K. Arai, J. Magn. Magn. Mater. 290, 1318 (2005)

14. L. Ding, S. Saez, C. Dolabdjian, L.G.C. Melo, A. Yelon, D. Ménard, IEEE Sens. J. 9, 159 (2009)

15. B. Dufay, S. Saez, C. Dolabdjian, A. Yelon, D. Ménard, IEEE Sens. J. 13, 379 (2013)

16. M. Malatek, B. Dufay, S. Saez, C. Dolabdjiana, Sens. Actuators A 204, 20 (2013)

17. E. Fernández, A. García-Arribas, M. Barandiarán, A.V. Svalov, G.V. Kurlyandskaya, C.P. Dolabdjian, IEEE Sens. J. 15, 6707 (2015)

18. L.G.C. Melo, D. Ménard, A. Yelon, L. Ding, S. Saez, C. Dolabdjian, J. Appl. Phys. 103, 033903 (2008) 